\title{
Whole-Genome sequencing and comparative genomics of Mycobacterium spp. from farmed Atlantic and coho salmon in Chile
}

\author{
Rudy Suarez $\cdot$ Karina Kusch $\cdot$ Claudio D. Miranda $\cdot$ Tianlu Li $\cdot$ \\ Javier Campanini · Phani Rama Krishna Behra • Luis Aro • Alexis Martínez • \\ Marcos Godoy • Daniel A. Medina
}

Received: 27 November 2020/ Accepted: 8 May 2021/Published online: 30 May 2021

(C) The Author(s) 2021, corrected publication 2021

\begin{abstract}
Several members of the Mycobacterium genus cause invasive infections in humans and animals. According to a recent phylogenetic analysis, some strains of Mycobacterium salmoniphilum (Msal), which are the main culprit in bacterial outbreaks in freshwater fish aquaculture, have been assigned to a separate branch containing Mycobacterium franklinii ( $M$ fra), another species that causes
\end{abstract}

Supplementary information The online version contains supplementary material available at (https://doi.org/10.1007/ s10482-021-01592-w).

R. Suarez · K. Kusch · M. Godoy

Centro de Investigaciones Biológicas Aplicadas, Diego de

Almagro Norte 1013, Puerto Montt, Chile

R. Suarez

Programa de Magíster en Acuicultura, Facultad de Ciencias del Mar, Universidad Católica del Norte,

Larrondo 1281, Coquimbo, Chile

R. Suarez · A. Martínez

Cardonal S/N, AquaChile, Puerto Montt, Chile

C. D. Miranda

Laboratorio de Patobiología Acuática, Departamento de Acuicultura, Universidad Católica del Norte, Larrondo 1281, Coquimbo, Chile

T. Li

Epigenetics and Immune Disease Group, Josep Carreras Leukaemia Research Institute, Badalona, Barcelona, Spain infections in humans. However, this genus is little studied in an aquaculture context. Here, we isolated four Mycobacterium spp. strains from freshwater cultures of Atlantic and coho salmon in Chile and performed whole-genome sequencing for deep genomic characterization. In addition, we described the gross pathology and histopathology of the outbreaks. Several bioinformatic analyses were performed using the genomes of these four Mycobacterium isolates in conjunction with those of Msal strains, four Msal-like strains, and one Mfra strains, plus 17 other publicly

\author{
J. Campanini \\ Facultad de Medicina y Ciencia, Universidad San \\ Sebastián, Lago Panguipulli 1390, Puerto Montt, Chile \\ P. R. K. Behra \\ Department of Cell and Molecular Biology, Biomedical \\ Centre, Box 596, 75124 Uppsala, Sweden \\ L. Aro \\ Benchmark Genetics, Santa Rosa 560 oficina 25 B, \\ Puerto Varas, Chile \\ M. Godoy \\ Programa Cooperativo Doctorado en Acuicultura, \\ Universidad Católica del Norte, Larrondo 1281, \\ Coquimbo, Chile
}


available Mycobacterium genomes. We found that three isolates are clustered into the Msal branch, whereas one isolate clustered with the Mfra/Msal-like strains. We further evaluated the presence of virulence and antimicrobial resistance genes and observed that the four isolates were closely related to the Msal and Msal-like taxa and carried several antimicrobial resistance and virulence genes that are similar to those of other pathogenic members of the Mycobacterium clade. Altogether, our characterization Msal and Msal-like presented here shed new light on the basis of mycobacteriosis provides quantitative evidence that Mycobacterium strains are a potential risk for aquaculture asetiological agents of emerging diseases, and highlight their biological scopes in the aquaculture industry.

Keywords Aquaculture - Comparative genomics . Emerging pathogens $\cdot$ Mycobacterium . Wholegenome sequencing $\cdot$ Chile

\section{Introduction}

Mycobacteriosis refers to infections caused by some members of the bacterial genus Mycobacterium. In fish, mycobacteriosis is characterized as a chronic progressive disease, with several external signs such as emaciation, inflammation of the skin, exophthalmia, ulceration, and open lesions (Austin and Austin 2016). Commonly, Mycobacterium are found in a wide range of environmental niches, particularly in aquatic environments, and only a small percentage of these species cause diseases in humans and animals. Fish mycobacteriosis may take several years to progress from an asymptomatic state to clinical illness and can affect more than 150 fish species, both farmed and wild (Decostere et al. 2004; Gauthier and Rhodes 2009; Heckert et al. 2001; Zanoni et al. 2008). For example, Mycobacterium pseudoshottsii has recently been identified as the etiological agent of outbreaks in three farmed fish species (Dicentrarchus labrax,

M. Godoy · D. A. Medina $(\square)$

Laboratorio de Biotecnología Aplicada, Facultad de Medicina Veterinaria, Universidad San Sebastián, Lago Panguipulli 1390, Puerto Montt, Chile

e-mail: daniel.medina@uss.cl
Sparus aurata, and Sciaenops ocellatus), thus indicating that various farmed fish species are affected by Mycobacterium (Mugetti et al. 2020).

Currently, more than 170 distinct species have been identified within the Mycobacterium genus (Forbes 2017), and three of these, namely M. marinum (Mma), M. fortuitum (Mfor), and M. chelonae (Mche), have been recognized as major causes of mycobacteriosis in fish (Gauthier and Rhodes 2009; Gcebe et al. 2018; Heckert et al. 2001; Rhodes et al. 2001; Whipps et al. 2008; Talaat et al. 1999). The typical gross pathology of mycobacteriosis in fish includes non-specific phenotypic changes, such as lethargy and anorexia, cutaneous ulcers, and decolouration (Bruno et al. 1998; Brocklebank et al. 2003; Luo et al. 2018). Several case studies have identified the presence of multiple granulomas in the skin and internal organs (Bruno et al. 1998; Brocklebank et al. 2003; Luo et al. 2018; Keller et al. 2018).

Recently, the list of species causing piscine mycobacteriosis has been expanded to include Msal, a taxonomically controversial specie. Msal was first classified in 1960 following its isolation from the kidneys of salmonid fishes and was described as an acid-fast bacillus (Ross 1960). However, owing to its biochemical similarities with M. fortuitum (Gordon and Mihm 1959) and the fact that two species could not be distinguished from each other, Msal was subsequently omitted as a separate Mycobacterium species (Skerman et al. 1989). Thereafter, following subsequent advances in molecular techniques, Whipps et al. (2007) have performed a phylogenetic analysis of the SSU rRNA genes of the strains isolated from salmonid fishes and confirmed that Msal is a monophyletic species, which is included within the McheM. abscessus (Mabs) complex (MCAC), which contains many clinically relevant human pathogens but Msal has not been implicated as a cause of disease in humans (Simmon et al. 2011).

Advances in molecular techniques allowed genomic analysis with single-nucleotide resolution and thus propelled the characterization and identification of Msal. Zerihun et al. (2011a) were the first to identify an Msal strain isolated from infected Norwegian salmon. By aligning the partial sequences of the $16 \mathrm{~S}$ rRNA, rpoB, and $H s p 65$ genes, the isolated strain was found to be 95-99\% similar to Msal ATCC 13758. Later, Aro et al. (2014) utilized a similar strategy to identify the strain they isolated from an infected 
Chilean Salmo salar, and they showed that the $16 \mathrm{~S}$ rRNA gene sequence obtained from Msal isolates shared $100 \%$ similarity with those from the Msal DQ866770 and Msal DQ866766 strains. In addition, these studies have found that the infected fish displayed significant macroscopic symptoms, such as external ulcers, poor body condition, pale gills, and erosion in the pectoral fin. Internal lesions, loss of adipose tissues, liver discolouration, and swelling of the internal organs have also been reported (Zerihun et al. 2011b, a; Aro et al. 2014). Nevertheless, both studies lacked of a genome-wide characterizations of the isolated strains. whole-genome.

The ability of Msal to infect mammals was first tested in vitro by Harriff et al. (2008). They observed that several Msal strains could infect and grow in both mouse and human macrophage cell lines. More recently, Msal infection has been reported in mice, in which an outbreak in an animal facility at Uppsala University was confirmed through deep DNA sequencing to have been caused by Mycobacterium species (Behra et al. 2019). The authors identified one as Msal, and the other three strains as Msal-like, which they proposed as new species of Mycobacterium after performing comparative genomics with 36 other MCAC members. Another recent study detected the presence of Renibacterium salmoninarum and $M y$ cobacterium spp. in wild brown trout in Austria during summer. Owing to the importance of $R$. salmoninarum in aquaculture as an etiological agent of bacterial kidney diseases, the presence of both pathogens in wild fish could present a potentially serious infectious disease risk for the aquaculture industry (Delghandi et al. 2020).

The objective of this study was characterizing the macroscopic and microscopic traits of mycobacteriosis and performing whole-genome sequencing on the four Mycobacterium strains isolated from S. salar and Oncorhynchus kisutch salmon aquaculture facilities, presenting both kind of results, in silico and in vivo findings simultaneously that further describe the pathogenicity of mycobacteriosis. In addition, we compared the genomic and functional features of these strains with those found in several publicly available Mycobacterium genomes. In overall, this study gives novel insight regarding the identification and deep characterization of Mycobacteryum isolates using a combination of several macroscopic, microscopic, phenotypic, and genomic approaches.

\section{Materials and methods}

Ethical statement

Tissue sampling and fish manipulation was performed under the Guidelines for the Use of Fishes in Research (Nickum 2004) and the Care and Use of Fish in Research, Teaching and Testing (Canadian Council on Animal Care 2005).

Strain isolation, cultivation, and characterization

Three mycobacterial strains (myc161, myc162 and myc182) were isolated from $S$. salar and one (myc151) was isolated from $O$. kisutch, both of which were obtained from freshwater farms located in the IX and X regions of Chile between 2015 and 2018 (Table 1). The bacterial isolates were recovered following the method described by Zerihun et al. (2011a, b). Briefly, bacteria were recovered from the livers of infected fishes in which grey-white nodules were present. Pieces of infected tissue were carefully disaggregated and suspended in sterile phosphatebuffered saline (PBS). Volumes of $1 \mathrm{~mL}$ of serial dilutions (ranging from $10^{-5}$ to $10^{-7}$ of the initial solution concentration) were plated on tryptic soy agar medium (TSA; Becton Dickinson, USA), and plates were incubated at $25^{\circ} \mathrm{C}$ for 5 to 10 days (Austin and Austin 2016). White creamy, brilliant, slightly convex colonies were subsequently transferred to modified Anacker and Ordal's agar (MAOA) medium as previously reported (Zerihun et al. 2011b; Aro et al. 2014). Growth sensitivity to temperature was evaluated by culturing the isolates at 16,25 , and $37{ }^{\circ} \mathrm{C}$ for 5 days on solid TSA, MacConkey agar, brain and heart infusion agar (BHI), MAOA, blood agar (BA), and TSA supplemented with $5 \% \mathrm{NaCl}$. Biochemical characteristics of the strains were determined using the Analytical Profile Index (API) 20E® system (BioMérieux, France) following the manufacturer's instructions.

\section{Histopathologic analysis}

Tissue samples from the gills, heart, liver, kidney, spleen, stomach, intestines, and pyloric caeca were collected from euthanised fish and fixed in $10 \%$ buffered formalin to prepare them for analysis. The fixed tissues were processed as previously described 
Table 1 Descriptions of the four strains

\begin{tabular}{lllllllll}
\hline Strain & Region & Recovered from & Year & Life stage & water & weight $(\mathrm{g})$ & Gross pathology & associated diseases \\
\hline myc161 & IX & S. salar & 2016 & Smolt & River & 3500 & Yes & No \\
myc182 & X & S. salar & 2018 & Fry & Watershed & 80 & No & No \\
myc162 & X & S. salar & 2016 & Broodstock & River & 3600 & Yes & No \\
myc151 & X & O. kisutch & 2015 & Fry & Lake & 100 & No & Flavobacteriosis \\
\hline
\end{tabular}

(Prophet 1992), and paraffin-embedded samples were sectioned into 3- to 4- $\mu \mathrm{m}$ blocks. The sections were mounted on glass slides and stained with haematoxylin and eosin according to previously described protocols (Fischer et al. 2008). Microscopic examination was carried out using a Leica DM2000 microscope (Leica, Germany) and visualized utilizing LAS software V3.3-2019 (Leica).

DNA isolation and sequencing

Bacterial DNA extraction was performed using a modified protocol consisting of phenol chloroform and tissue homogenization using glass beads (Anahtar et al. 2016). To facilitate bacterial wall rupture, enzymatic digestion was performed with lysozymes at $37{ }^{\circ} \mathrm{C}$ for $1 \mathrm{~h}$, followed by Proteinase $\mathrm{K}$ incubation at $55{ }^{\circ} \mathrm{C}$ overnight prior to bead-beating homogenization (Gill et al. 2016). DNA integrity was analysed using $1 \%$ agarose gel electrophoresis, and nucleic acid quantification was performed using a NanoDrop Lite (Thermo Scientific, USA). To confirm that the isolates belong to the Mycobacterium genus, a PCR assay was performed using the $27 \mathrm{~F}$ and $1492 \mathrm{R}$ primers for the amplification of the 16S rRNA gene (Weisburg et al. 1991). PCR products were analysed using Sanger sequencing with an ABI PRISM 3500 XL sequencer (Applied Biosystems, USA), and the obtained sequences were used for BLAST analysis (Altschul et al. 1990). For whole-genome sequencing, $2 \mu \mathrm{g}$ DNA was sequenced by the Novogene Corporation Inc. (Sacramento, USA) on an Illumina Novaseq 6000 platform using $2 \times 150$ paired-end. The raw data reads obtained from the Illumina platform have been deposited at the ENA-EMBL database under the accession number PRJEB38737 (Table 2).
Quality control, assembly, and genome annotation

Raw sequences generated in this study and those from a previous report (Behra et al. 2019) were preprocessed using Trimmomatic v0.39 to remove the leading and trailing bases with quality $<28$ and drops reads $<120$ bases long. Bases with average quality scores below 20 were trimmed (Bolger et al. 2014). The filtered reads were mapped against the Msal DSM43276 genome using SMALT aligner v0.7.6 available at the Sanger Institute (https://www.sanger. ac.uk/science/tools/smalt-0). SAMtools and BCFtools (http://www.htslib.org) were used to generate the consensus FASTA sequences ( $\mathrm{Li}$ et al. 2009). The genomes were annotated using Prokka v1.12 (Seemann 2014), and the core- and pan-genomes were assessed using Roary v3.12.0 with a minimum percentage identity of $95 \%$ for blastp (Page et al. 2015). The core- and pan-genome were drawn using the script roary2svg.pl as described in the Roary pan-genome pipeline (https://sanger-pathogens.github.io/Roary/).

\section{Comparison of Mycobacterium genomes}

Full-length 16S rRNA sequences from our four newly sequenced genomes and from 30 additional mycobacterial genomes retrieved from the NCBI database (Supplementary Table 1) were extracted using metaxa2 v2.2.1 (Bengtsson-Palme et al. 2015), and phylogenetic dendrograms were constructed using the Decipher package (Wright 2016) in $\mathrm{R}$ environment (R Core Team 2013). The full-length $r p o B$ gene sequences obtained from the Prokka annotation were used for phylogenetic classification. The evolutionary distance at the species level was assessed using average nucleotide identity (ANI) analysis of the homologous genomic regions, and the 28 publicly available Mycobacterium complete genome sequences 
Table 2 Summary of genome annotations, coding sequences, and accession number of whole-genome sequences produced in this study

\begin{tabular}{lllllll}
\hline Isolate & Reads & GC content $(\%)$ & Assembly size $(\mathrm{bp})$ & Coverage & No. CDS & ENA Accesion n. \\
\hline myc161 & $14,727,378$ & 64 & $4,582,212$ & 964 & 4434 & ERS4631745 \\
myc182 & $15,638,477$ & 63 & $4,391,176$ & 1068 & 4277 & ERS4631746 \\
myc162 & $15,366,177$ & 63 & $4,371,854$ & 1054 & 4268 & ERS4631747 \\
myc151 & $17,400,957$ & 64 & $3,993,798$ & 1307 & 4080 & ERS4631748 \\
\hline
\end{tabular}

were compared using pyani module v0.2.10 (Pritchard et al. 2016). For this purpose, genomes were aligned using the MUMmer tool v3.23 (Kurtz et al. 2004).

Functional annotation of orthologous genes

The sequences previously obtained from the Prokka analysis were annotated against NCBI's cluster of orthologous groups (COG) database using the web tool WebMGA (Wu et al. 2011), and orthologous clustering was subsequently performed using OrthoVenn2 (Xu et al. 2019) (https://orthovenn2. bioinfotoolkits.net/). Unique elements were retrieved for orthologous classification from OrthoVenn2, and their sequences were used for protein identification in Blastp (Altschul et al. 1990). The virulence and antimicrobial resistance genes were identified using ABRIcate v0.9.8 (https://github.com/tseemann/ abricate) by means of the NCBI Bacterial Antimicrobial Resistance Reference Gene Database (Feldgarden et al. 2019), Resfinder (Zankari et al. 2012), and the Virulence Factors of Pathogenic Bacteria database (VFDB) (Chen et al. 2016).

\section{Results}

Anatomopathological and microscopic findings

Three Mycobacterium strains were isolated from $S$. salar and $O$. kisutch, respectively, that were obtained from freshwater farms in the south of Chile. Macroscopically, we found raised scales in both salmonid species, as shown for $S$. salar (Fig. 1A). Internally, the fish presented hepatomegaly, splenomegaly, and posterior renomegaly. In the liver, the presence of pseudomembranes with opaque colouration was recurrent. Histopathologically, the fish presented multiple foci of mononuclear cells in the liver and variable infiltration of mononuclear cells in the liver and spleen parenchyma. In both species, food was not detected in the digestive system of the diseased fish.

Specifically, the mycobacteriosis on S. salar was characterized by pale gills with multiple white nodules. Internally, we also observed the accumulation of visceral fat with signs of hepatomegaly, renomehalia, splenomegaly, and variably shaped white nodules in the kidney, liver, and spleen (Fig. 1B). Histologically, we observed multiple granulomas composed of mononuclear cells, surrounded by fibroblasts, giant cells, and multifocal granulomatous nephritis (Fig. 2a).

There were some stark differences with the mycobacteriosis in $O$. kisutch. Mainly, inner peritonitis was observed (Fig. 1c). Internally, the fish showed hepatomegaly, splenomegaly, posterior renomegaly, accumulation of visceral fat, ascites, and the presence of pseudo-membranes in the liver and spleen (Fig. 1c and d). Histopathologically, in the liver, multiple foci of mononuclear cells (Fig. 2b) and fibrin deposits in its capsule were detected, consistent with subacute to chronic multifocal hepatitis. In addition, the infiltration of mononuclear cells in the splenic and renal parenchyma was observed, which indicate diffused, subacute, and mild to moderate nephritis and splenitis. Also, cutaneous flavobacteriosis infection symptoms were observed in this species.

\section{Microbiological characterization}

After 5 days of incubation in the MAOA medium, we obtained pure white, circular, smooth and well-defined colonies (Supplementary Fig. 1a). Ziehl-Neelsen staining results revealed that all strains were rod acid-fast bacteria (Supplementary Fig. 1b) similar to 


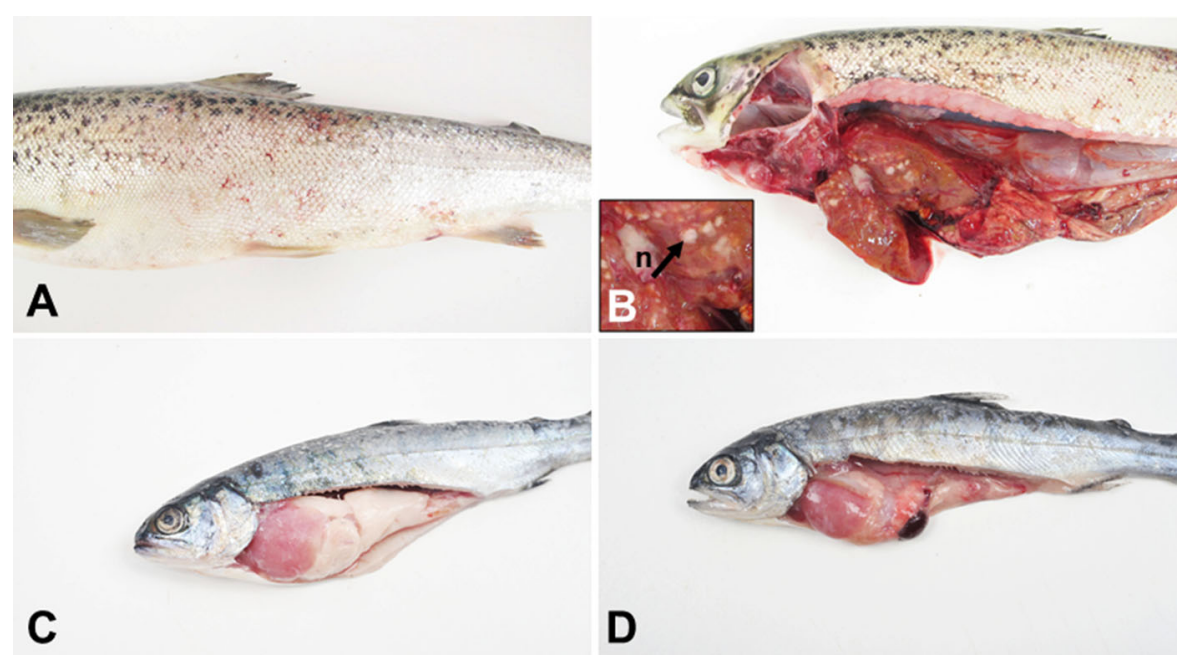

Fig. 1 Macroscopic findings of mycobacteriosis. (a) Skins lesion on S. salar. (b) internal overview of S. salar. (c \& d) O. kisutch internal overview
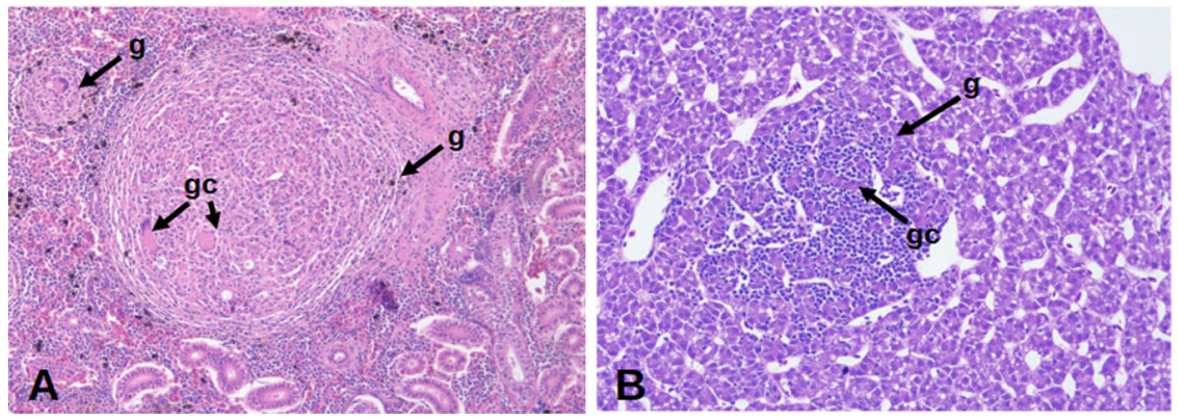

Fig. 2 (a) Kidney and liver (b) H\&E granulomas at $40 \times$. Arrows indicate white nodules (n), granulomas (g), and giant cells (gc)

those observed in the kidney smear (Supplementary Fig. 1c).

To initially characterize the bacteriological phenotypes of the strains, their growth sensitivity to temperature was tested (Aro et al. 2014). The optimal growth of the Mycobacterium strains was obtained at $25{ }^{\circ} \mathrm{C}$ in all media used, and a decreased growth rate was observed at $16{ }^{\circ} \mathrm{C}$. However, incubation at $37{ }^{\circ} \mathrm{C}$ resulted in complete growth inhibition. Biochemical characterization using the Analytical Profile Index (API) 20E® system (BioMérieux, France), showed that all the isolates were positive for urease activity and glucose fermentation, and no other enzymatic activities were detected.
Genomic features

PCR amplification and fragment sequencing of the 16S rRNA gene (Anahtar et al. 2016) revealed that the four isolates belonged to the Mycobacterium genus (Supplementary Table 2). Whole-genome sequencing results showed that the average number of reads was $15,783,247$, and the average size of the genomes was $4,334,760$ base pairs. The mean GC-content of the four strains was $63 \%$, and the annotated predicted proteins were obtained from an average of 4265 coding sequences (Table 2).

A BLAST search using the full-length 16S rRNA sequences retrieved from the whole-genome sequences confirmed that the bacterial strains recovered from S. salar (myc161, myc182, and myc162) belonged to the species Msal (Supplementary Table 2), whereas the strain isolated from $O$. kisutch (myc151) 
was identified as Mche. Subsequently, we used the same approximation described using the other 30 Mycobacterium whole-genome sequences, which were publicly available in the NCBI nucleotide database(Supplementary Table 1), to construct phylogenetic trees based on full-length 16S rRNA gene sequences using the tools provided by the $\mathrm{R}$ package Decipher (Wright 2016). In the phylogenetic tree constructed, the isolates myc161, myc182, and myc162 are clustered in the Msal branch, whereas the myc151 are clustered with the previously characterized Msal-like strains (Behra et al. 2019) and in close proximity to $M$ fra and some Mche strains. In parallel, $M$. fortuitum, M. lepraemurium, M. avium, $M$. paraintracellulare, M. tuberculosis, Mma, and two different Mche strains are clustered in a separate phylogenetic branch (Fig. 3). Identical results were obtained using the full-length $r р о B$ sequences obtained from whole-genome annotations (Supplementary Fig. 2).

\section{Average Nucleotide Identity (ANI)}

To understand the interrelationship between the four Mycobacterium strains and other Mycobacterium species, we performed unsupervised clustering aided by ANI analysis (Konstantinidis and Tiedje 2005) using a total of 34 Mycobacterium whole-genome sequences, including 30 previously published genomes (Supplementary Table 1). First, the four isolates were clustered with genomes belonging to the Msal, Msal-like, Mfra, Mabs, and Mche species, yielding ANI scores $>50 \%$ and forming five related clusters. Seven genomes belonging to M. fortuitum, Mche, $M$. tuberculosis, Mma, M. lepraemurium, M. avium, and M. paraintracellulare clustered separately with ANI scores $<25 \%$ (Fig. 4). Second, ANI alignment coverage revealed that myc162, myc182, and myc161 were clustered together with the Msal genomes. However, myc151 was clustered with the genomes of the Msal-like strains and those of some Mfra strains, in accordance with the results obtained using the fulllength 16S rRNA and rpoB sequences (Fig. 3 and Supplementary Fig. 2), thereby indicating that this strain does not correspond to Msal.

Finally, according to the $95 \%$ identity threshold (Pritchard et al. 2016), myc162, myc182, and myc161 were most similar in identity to the Msal genomes, whereas myc151 potentially belongs to the Mfra and
Msal-like species, a genetically separate phylogenetic branch (Behra et al. 2019).

Comparative analysis of core- and pan-genomes

The annotated assemblies obtained by the Prokka tool (Seemann 2014) from the 27 whole-genomes belonging to the Msal, Msal-like, Mfra, Mabs, and Mche species, were then used to calculate the core-genome and pan-genome, in which gene presence for each genome was represented in parallel to compare similarities between species (Supplementary Fig. 3). Overall, annotated gene number ranged from 4,071 to 5,396, with myc151 and Mfra-DSM45524 containing the least and the greatest number of genes, respectively. Genome analysis of the Mycobacterium species yielded 23,935 predicted genes, of which 861 genes were shared across all species and comprised the core genome.. Furthermore, after comparing the gene content patterns of the isolates, we found that myc182 and myc162 displayed the greatest core similarities to Msal strains CCUG60883, CCUG60885, DE4585, and DE4587, whereas myc161 shared core patterns with the genomes of Msal CCUG60884, CCUG62472, and DSM43276 (Supplementary Fig. 4). Lastly, myc151 shared a core pattern with Mfra and the Msal-like species, supporting the results obtained via the $16 \mathrm{~S}$ rRNA phylogeny and ANI analysis (Figs. 2, 4 and Supplementary Fig. 4).

Functional classification of annotated genes

A protein function clustering of the 4 Chilean isolates utilizing the COG database (Tatusov 2000) was performed to describe the functional patterns of annotated genes. All four isolates displayed almost identical functional categories, being the "R: general functional prediction only" and "S: function unknown" (Fig. 5a) the most enriched COG categories. The other functional COG categories included transcription, secondary metabolites biosynthesis, transport, and catabolism (categories $\mathrm{K}$ and Q). Subsequently, a differential analysis of orthologous gene clusters between isolates was conducted, and we found that the four strains formed 4,317 clusters of orthologs, including 3,473 core gene orthologs and 4 unique elements without orthologs (Fig. 5b). Interestingly, those belonging specifically to myc151, myc161 


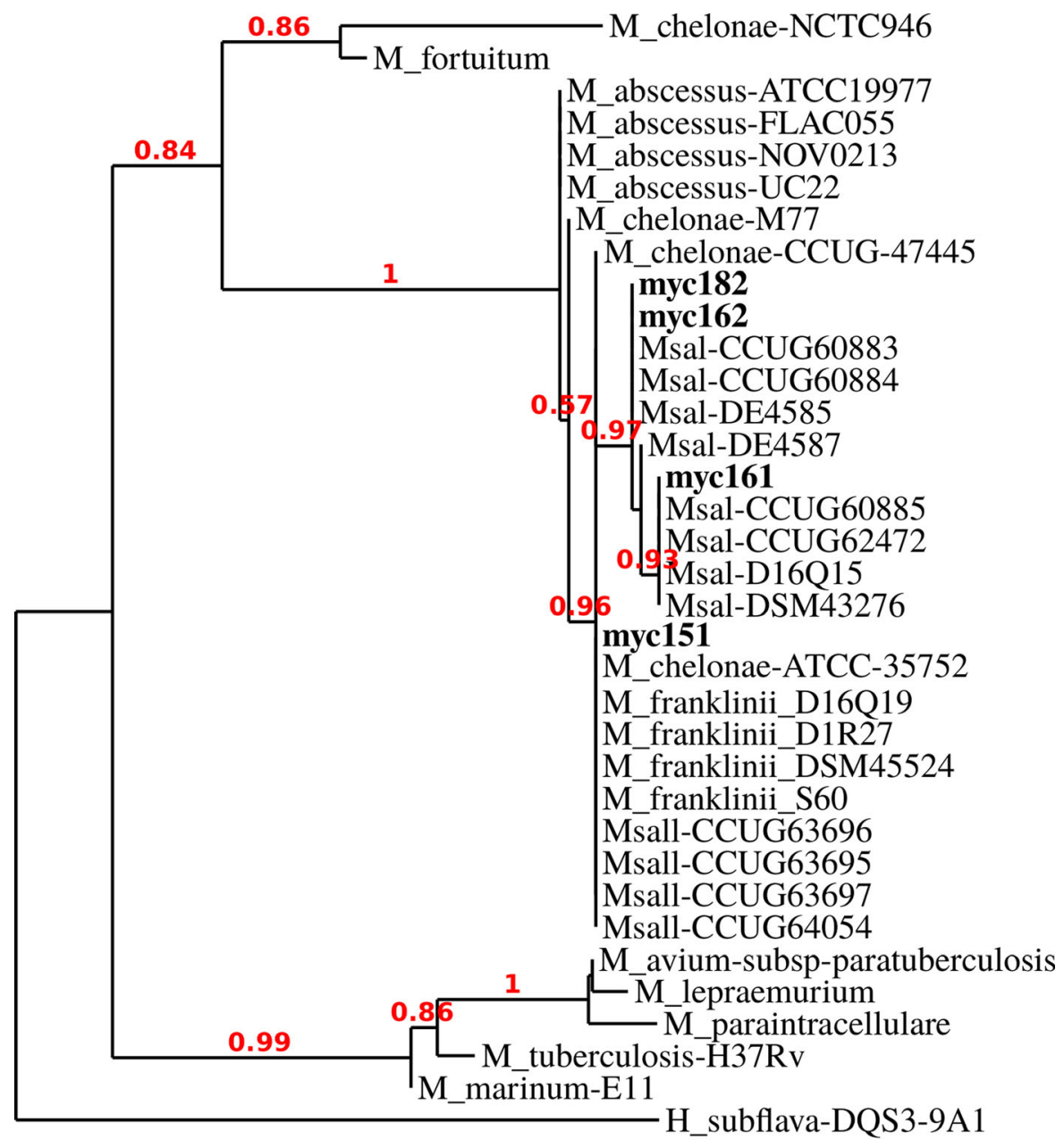

\subsection{3}

Fig. 3 Dendrogram based on the phylogenetic relationships of the $16 \mathrm{~S}$ rRNA gene sequences. Sequences were retrieved from the whole-genome sequencing of 4 Chilean isolates (in bold letters) and compared with 30 other Mycobacterium species

and myc182, were already described for some Mycobaterium isolates. The unique element for myc161 was predicted to be a hypothetical protein, that had been previously predicted in Mche (NCBI Acc. Number: WP_200996060). For myc151, one element matched with a PE family protein annotated in Msal (WP_134081245) and Mfra (WP_134048526), and the second element was predicted to be a hypothetical protein, annotated in Mabs (NCBI Acc. Number: SKT85326). The unique element belonging to myc 182 was predicted to be a family type VII secretion target that was previously identified in Mfra obtained from Behra et al. (2019) and the NCBI database. The Hoyosella subflava DQS3-9A1 sequence was used as an outgroup, and bootstrap values are denoted in red

(WP_070937634) and Msal (WP_134062353). Altogether, these observations suggest that the unique elements identified are not strain specific, and instead belong more broadly to the Mycobacterium clade.

Identification of virulence and antimicrobialresistance genes

Next, we surveyed the whole-genome of the four isolates for the presence of genes encoding virulence factors (VF) and antibiotic resistance (AR). The isolates shared the presence of several VF genes, 


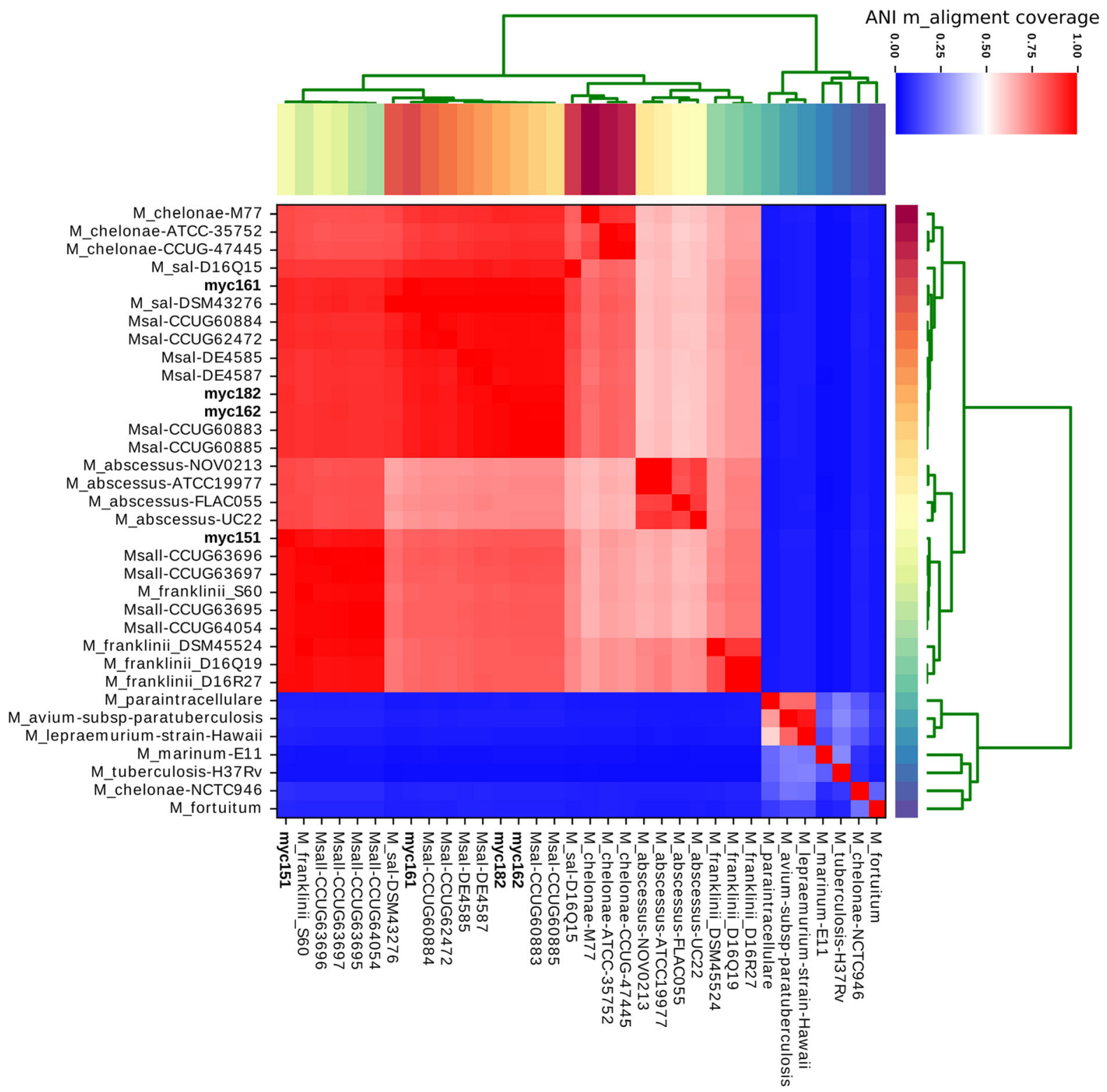

Fig. 4 Average Nucleotide Identity (ANI) based on the wholegenome sequences of 34 genomes belonging to the Mycobacterium genus. Clustering of the whole genomes was based on the MUMmer alignment of the input sequences and is represented by the heatmap. The ANI m_aligment coverage represents the

including $i d e R, i c l, m b t H, r e l A$, and phoP. Interestingly, $m b t H$ encodes the siderophore protein mycobactin (VF0299), which is involved in the shuttling of free extracellular iron ions into the cytoplasm of mycobacterial cells (Supplementary Fig. 5). Besides, $i d e R$ also regulates functions related to iron metabolism, suppressing genes that encode proteins involved percentage of nucleotide identity in the matching regions among genomes (coloured blue for low coverage and red colour for high alignment coverage). The horizontal tree depicts the clustering of column-wise dendrogram

in siderophore synthesis (mbtA-G), secretion (mmpL4/5, mmpS4/5) and uptake (irtAB) (Zondervan et al. 2018).

Regarding AR genes, only the strains myc162 and myc182 encoded genes associated with the resistance to carbapenem, such as CRP-1 and carbapenemhydrolysing class A beta-lactamase. This result was 

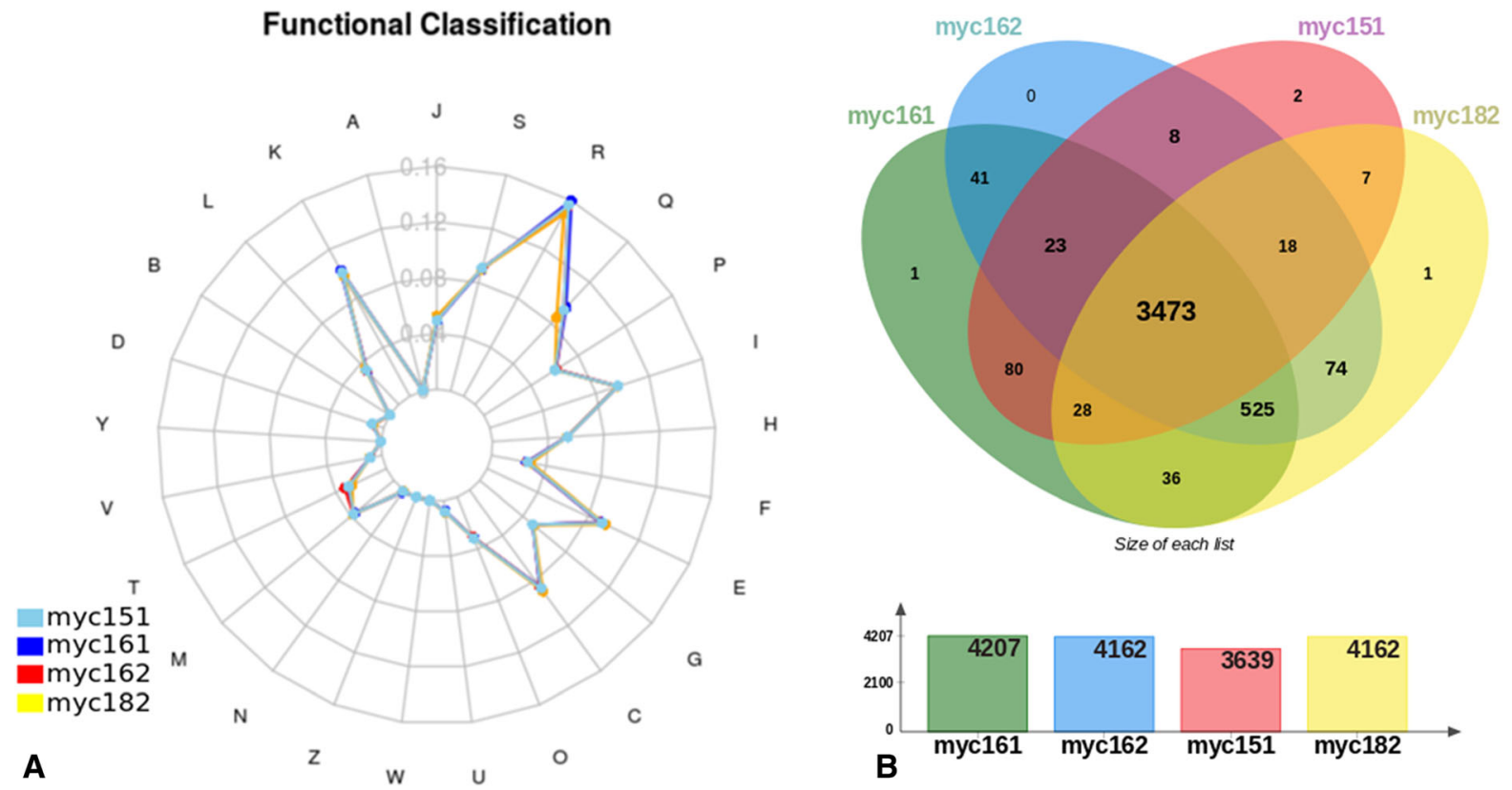

Fig. 5 Functional classification of annotated genes of $M y$ cobacterium isolates from Chile. (a) Spider web diagram of the abundance profile of Clusters of Orthologous Groups (COGs)

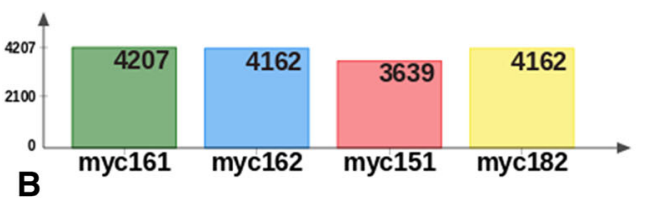

consistent with the high amount of genetic similarity of myc162, myc182, and some Msal strains, specifically Msal CCUG60883, CCUG60885, DE4585, and DE4587, which also exhibit resistance to carbapenem. Finally, we showed that the other analysed Mycobacterium strains possessed genes for resistance to betalactam, tetracycline, gentamycin, macrolide, and rifampin (Supplementary Table 3).

\section{Discussion}

This study comprehensively analysed the genomes of serveral Mycobacterium strains. The presence of this genus had been previously reported years ago in Chile (Aro et al. 2014). Here, we expand a previous the report of mycobacteriosis in Chile, characterising the whole-genome of 4 isolates derived from salmon freshwater aquaculture facilities. This new description includes whole-genome analyses of four isolated bacterial strains, three of which (myc161, myc182 and myc162) were from $S$. salar, and one of which (myc151) was from $O$. kisutch. All four were isolated from freshwater systems, which suggests that these Mycobacterium strains specifically affect the

identified from whole-genome sequencing. (b) Venn diagram of the comparative analysis between Chilean isolates showing the common and unique annotated predicted genes

freshwater stage of salmonid farming. First, macroscopically, the fish displayed biological characteristics consistent with previous descriptions of infections caused by members of the Mycobacterium genus(Aro et al. 2014; Bruno et al. 1998; Brocklebank et al. 2003; Keller et al. 2018; Luo et al. 2018; Parikka et al. 2012). Second, our bacteriological findings and 16S rRNA PCR results confirmed that the isolates belong to the Mycobacterium clade. Whole-genome sequencing confirmed that three of the isolates: myc161, myc182, and myc162, belonged to the Msal species, while the isolate myc151 was closely related to the new Mfra/Msal-like phylogenetic branch previously described by Behra et al. (2019), wherein the authors identified significant genomic differences between the strains. As such, these Msal-like strains belong to a single species according to their ANI score.

In accordance with previously reported data, we observed that the Mfra strains displayed patterns that were similar, but not identical, to those of the Msallike strains in terms of their 16S rRNA, wholegenome, and core- and pangenome sequences as the Msal-like strains. This supports the existence of an independent $\mathrm{Mfra} / \mathrm{Msal}$-like species closely related to Mfra. Interestingly, only the strain isolated from coho 
salmon was identified as belonging to the MfralMsallike species, whereas those from the Atlantic salmon were identified as Msal (Figs. 3, 4 and Supplementary Fig. 2).

The bacterium Mfra was first classified as a member of the Mche-Mabs complex (MCAC) by Simmon et al. (2011), who isolated it from patients with underlying lung conditions. Although the exact source of the disease-causing pathogen is unknown, another study (Van Ingen et al. 2010) has detected Mfra in municipal water sources, suggesting an environmental origin that may be region-specific. Collectively, these studies suggest that terrestrial $\mathrm{Mfra} / \mathrm{Msal}$-like infections may directly originate from nearby water sources. Hence, this is the first study to report a newly proposed species, which potentially cause mycobacteriosis in salmonids, belonging to the Mfra/Msal-like cluster from a Chilean freshwater breeding system, highlighting the need to monitor these mycobacteriosis-related diseases in aquaculture settings. On the other hand, $M s a l$ has been previously reported to cause mycobacteriosis outbreaks in S. salar and other fish species (Whipps et al. 2007; Zerihun et al. 2011b, a; Austin and Austin 2016). However, no evidence has thus far suggested that Msal causes infections in terrestrial animals. Interestingly, the mycobacteriosis described here manifested differently in coho and Atlantic salmon species. Mycobacteriosis in S. salar was characterized by internal granulomas, whereas $O$. kisutch mainly exhibited chronic peritonitis (Fig. 1). Nevertheless, we cannot eliminate the possibility that different Mycobacterium species cause distinct disease manifestations.

Recent advances in sequencing technology facilitated the identification of several Mycobacterium species and subspecies based on phylogenetic algorithms from multiple genomic databases, thus generating more accurate and robust phylogenetic classification. One such example is Das et al. (2018), who analysed the genome sequences of $19 \mathrm{Mma}$ strains using comparative genomics, ANI analysis, and phylogenetic trees to cluster the strains into two distinct branches. Similarly, Behra et al. (2019) performed several comparative genomic analyses and concluded that Msal-like isolates and Mfra isolates are similar enough to constitute a single species. Recently, a study performed on isolates recovered from gilthead seabream identified Mma as an etiological agent of mycobacteriosis using molecular and mass spectrometry (MALDI-TOF) analyses (Davidovich et al. 2020). In this work, in addition to $16 \mathrm{~S}$ rRNA analysis for phylogenetical classification, we utilized multiple genomic tools to assign the identities and characterise the strains isolated. Altogether, this work highlights the importance of reviewing previous classifications of infectious Mycobacterium species using novel scientific tools and several bioinformatic approaches.

One limitation of our study was the low number of Chilean isolates analysed. However, the identification of new bacterial isolates from aquaculture facilities may confirm the presence of the Msal-like strains. Our findings pave the way for the further investigation and monitoring of this bacterial genus as a potential etiological agent of an emerging disease, namely mycobacteriosis in aquaculture systems. Interestingly, virulence factors were found in all the strains studied that have been reported in other mycobacterium spp.; phoP (Ryndak et al. 2008), mbtH (Baltz 2011), ideR (Zondervan et al. 2018), among others. These last two genes, regulate iron homeostasis through various mechanisms, and play a crucial role in mycobacterium tuberculosis virulence (Pandey and Rodriguez 2014). According to, this VFs has been used as an important target to guide the search for new drugs (Wang et al. 2016; Rohilla et al. 2017; Salimizand et al. 2017). If strategies are found that successfully inhibit these targets, they may be used to treat multiple other mycobacteriosis of human and animal origin. In addition, the presence of AR genes in myc162 and myc182 that encode genes for resistance to carbapenem, points to the need to study the minimum inhibitory antibiotic concentrations for these and other new isolates. Finally, our findings will serve as guide to future studies aiming to provide insights into mycobacterial biology and pathogenicity using a combination of several tools simultaneously.

\section{Declarations}

Authors' Contributions RS performed sample collection and provided information about the strains. CDM contributed to reviewing the manuscript. TL contributed to writing and revising the manuscript and discussing the results. JC performed the phenotypic, biochemical and microbiological characterizations of the isolates. LA and AM contributed with 
the mycobacterial isolates. KK performed the histopathology analysis. PB critically read the manuscript, provided some of the genomic Mycobacterium raw data for comparative genomics, and supervised the data analysis. MG supervised the study, conceptualized the experiments, described the gross pathology and histopathology associated with mycobacteriosis, and critically edited the manuscript. DM direct the study, conceived the experiments and analysis, performed the bioinformatic and comparative genomic analyses, analysed the data, and wrote the manuscript. All authors agreed that the manuscript is published.

Funding This study was funded by the Centro de Investigaciones Biológicas Aplicadas and supported by the Universidad San Sebastián, Facultad de Medicina Veterinaria, Sede de La Patagonia.

Availability of data and material The whole-genome datasets generated during the current study are available in the ENA-EMBL (https://www.ebi.ac.uk/ena/browser/view/ PRJEB38737), and their accession numbers are provided in Table 2 and Supplementary Table 1 . All other data generated or analyzed during this study are included in this published article and its supplementary information files.

\section{Declarations}

Conflicts of interest The authors declare that the research was conducted in the absence of any commercial or financial relationships that could be construed as a potential conflict of interest.

Open Access This article is licensed under a Creative Commons Attribution 4.0 International License, which permits use, sharing, adaptation, distribution and reproduction in any medium or format, as long as you give appropriate credit to the original author(s) and the source, provide a link to the Creative Commons licence, and indicate if changes were made. The images or other third party material in this article are included in the article's Creative Commons licence, unless indicated otherwise in a credit line to the material. If material is not included in the article's Creative Commons licence and your intended use is not permitted by statutory regulation or exceeds the permitted use, you will need to obtain permission directly from the copyright holder. To view a copy of this licence, visit http://creativecommons.org/licenses/by/4.0/.

\section{References}

Altschul SF, Gish W, Miller W et al (1990) Basic local alignment search tool. J Mol Biol 215:403-410. https://doi.org/ 10.1016/S0022-2836(05)80360-2

Anahtar MN, Bowman BA, Kwon DS (2016) Efficient nucleic acid extraction and 16S rRNA gene sequencing for bacterial community characterization. J Vis Exp 15:11-12. https://doi.org/10.3791/53939

Aro L, Correa K, Martínez A et al (2014) Characterization of Mycobacterium salmoniphilum as causal agent of mycobacteriosis in Atlantic salmon, Salmo salar L., from a freshwater recirculation system. J Fish Dis 37:341-348. https://doi.org/10.1111/jfd.12108

Austin B, Austin DA (2016) Bacterial fish pathogens: Disease of farmed and wild fish, sixth edition. Springer International Publishing

Baltz RH (2011) Function of MbtH homologs in nonribosomal peptide biosynthesis and applications in secondary metabolite discovery. J Ind Microbiol Biotechnol

Behra PRK, Das S, Pettersson BMF et al (2019) Extended insight into the Mycobacterium chelonae-abscessus complex through whole genome sequencing of Mycobacterium salmoniphilum outbreak and Mycobacterium salmoniphilum-like strains. Sci Rep 9:4603. https://doi.org/10.1038/ s41598-019-40922-x

Bengtsson-Palme J, Hartmann M, Eriksson KM et al (2015) metaxa2: Improved identification and taxonomic classification of small and large subunit rRNA in metagenomic data. Mol Ecol Resour 15:1403-1414. https://doi.org/10. 1111/1755-0998.12399

Bolger AM, Lohse M, Usadel B (2014) Trimmomatic: A flexible trimmer for Illumina sequence data. Bioinformatics 30:2114-2120. https://doi.org/10.1093/bioinformatics/ btu 170

Brocklebank J, Raverty S, Robinson J (2003) Mycobacteriosis in Atlantic salmon farmed in British Columbia. Can Vet $\mathrm{J}$ 44:486-489

Bruno DW, Griffiths J, Mitchell CG et al (1998) Pathology attributed to Mycobacterium chelonae infection among farmed and laboratory-infected Atlantic salmon Salmo salar. Dis Aquat Organ 33:101-109. https://doi.org/10. 3354/dao033101

Canadian Council on Animal Care (2005) Canadian Council on Animal Care guidelines on: the care and use of fish in research, teaching and testing. Can Counc Anim Care 1-94

Chen L, Zheng D, Liu B et al (2016) VFDB 2016: Hierarchical and refined dataset for big data analysis-10 years on. Nucleic Acids Res 44:D694-D697. https://doi.org/10. 1093/nar/gkv1239

Das S, Pettersson BMF, Behra PRK et al (2018) Extensive genomic diversity among Mycobacterium marinum strains revealed by whole genome sequencing. Sci Rep 8:1-15. https://doi.org/10.1038/s41598-018-30152-y

Davidovich N, Pretto T, Sharon G et al (2020) Cutaneous appearance of mycobacteriosis caused by Mycobacterium marinum, affecting gilthead seabream (Sparus aurata) cultured in recirculating aquaculture systems. Aquaculture. https://doi.org/10.1016/j.aquaculture.2020.735507

Decostere A, Hermans K, Haesebrouck F (2004) Piscine mycobacteriosis: a literature review covering the agent and the disease it causes in fish and humans. Vet Microbiol 99:159-166. https://doi.org/10.1016/j.vetmic.2003.07.011

Delghandi MR, Menanteau-Ledouble S, Waldner K, El-Matbouli M (2020) Renibacterium salmoninarum and Mycobacterium spp.: Two bacterial pathogens present at low levels in wild brown trout (Salmo trutta fario) populations in Austrian rivers. BMC Vet Res. https://doi.org/10. 1186/s12917-020-2260-7

Feldgarden M, Brover V, Haft DH et al (2019) Using the NCBI AMRFinder Tool to Determine Antimicrobial Resistance Genotype-Phenotype Correlations Within a Collection of 
NARMS Isolates. bioRxiv 550707. https://doi.org/10. $1101 / 550707$

Fischer AH, Jacobson KA, Rose J, Zeller R (2008) Hematoxylin and eosin staining of tissueand cell sections. Cold Spring Harb Protoc 3:pdb.prot4986. https://doi.org/10.1101/pdb. prot4986

Forbes BA (2017) Mycobacterial taxonomy. J Clin Microbiol. https://doi.org/10.1128/JCM.01287-16

Gauthier DT, Rhodes MW (2009) Mycobacteriosis in fishes: a review. Vet J 180:33-47. https://doi.org/10.1016/J.TVJL. 2008.05.012

Gcebe N, Michel AL, Hlokwe TM (2018) Non-tuberculous Mycobacterium species causing mycobacteriosis in farmed aquatic animals of South Africa. BMC Microbiol 18:32. https://doi.org/10.1186/s12866-018-1177-9

Gill C, Van De Wijgert JHHM, Blow F, Darby AC (2016) Evaluation of lysis methods for the extraction of bacterial DNA for analysis of the vaginal microbiota. PLoS ONE 11. https://doi.org/10.1371/journal.pone.0163148

Gordon RE, Mihm JM (1959) A comparison of four species of mycobacteria. J Gen Microbiol 21:736-748. https://doi. org/10.1099/00221287-21-3-736

Harriff MJ, Wu M, Kent ML, Bermudez LE (2008) Species of environmental mycobacteria differ in their abilities to grow in human, mouse, and carp macrophages and with regard to the presence of mycobacterial virulence genes, as observed by DNA microarray hybridization. Appl Environ Microbiol 74:275-285. https://doi.org/10.1128/AEM.01480-07

Heckert RA, Elankumaran S, Milani A, Baya A (2001) Detection of a new mycobacterium species in wild striped bass in the chesapeake Bay. J Clin Microbiol 39:710-715. https:// doi.org/10.1128/JCM.39.2.710-715.2001

Keller C, Wenker C, Jermann T, et al (2018) Piscine mycobacteriosis-involvement of bacterial species and reflection in pathology. Schweiz Arch Tierheilkd 160:385-393. https://doi.org/10.17236/sat00165

Konstantinidis KT, Tiedje JM (2005) Genomic insights that advance the species definition for prokaryotes. Proc Natl Acad Sci U S A 102:2567-2572. https://doi.org/10.1073/ pnas.0409727102

Kurtz S, Phillippy A, Delcher AL et al (2004) Versatile and open software for comparing large genomes. Genome Biol 5:R12. https://doi.org/10.1186/gb-2004-5-2-r12

Li H, Handsaker B, Wysoker A et al (2009) The Sequence Alignment/Map format and SAMtools. Bioinformatics 25:2078-2079. https://doi.org/10.1093/bioinformatics/ btp352

Luo Z, Li J, Zhang Z et al (2018) Mycobacterium marinum is the causative agent of splenic and renal granulomas in halfsmooth tongue sole (Cynoglossus semilaevis Günther) in China. Aquaculture 490:203-207. https://doi.org/10.1016/ j.aquaculture.2018.02.036

Mugetti D, Varello K, Gustinelli A et al (2020) Mycobacterium pseudoshottsii in mediterranean fish farms: New trouble for european aquaculture? Pathogens. https://doi.org/10.3390/ pathogens 9080610

Nickum J (2004) Guidelines for the use of fishes in research. Aquac Mag

Page AJ, Cummins CA, Hunt M et al (2015) Roary: Rapid largescale prokaryote pan genome analysis. Bioinformatics
31:3691-3693. https://doi.org/10.1093/bioinformatics/ btv421

Pandey R, Rodriguez GM (2014) IdeR is required for iron homeostasis and virulence in Mycobacterium tuberculosis. Mol Microbiol. https://doi.org/10.1111/mmi.12441

Parikka M, Hammarén MM, Harjula SKE et al (2012) Mycobacterium marinum Causes a Latent Infection that Can Be Reactivated by Gamma Irradiation in Adult Zebrafish. PLoS Pathog 8. https://doi.org/10.1371/journal. ppat. 1002944

Pritchard L, Glover RH, Humphris S et al (2016) Genomics and taxonomy in diagnostics for food security: Soft-rotting enterobacterial plant pathogens. Anal Methods 8:12-24. https://doi.org/10.1039/c5ay02550h

Prophet EB, Armed Forces Institute of Pathology (U.S.) (1992) Laboratory methods in histotechnology. American Registry of Pathology

R Core Team (2013) R Core Team. R A Lang Environ Stat Comput R Found Stat Comput Vienna, Austria ISBN 3-900051-07-0, URL http://www.R-project.org/.

Rhodes MW, Kator H, Kotob S et al (2001) A Unique $M y$ cobacterium Species Isolated from an Epizootic of Striped Bass ( Morone saxatilis ). Emerg Infect Dis 7:896-899. https://doi.org/10.3201/eid0705.017523

Rohilla A, Khare G, Tyagi AK (2017) Virtual Screening, pharmacophore development and structure based similarity search to identify inhibitors against IdeR, a transcription factor of Mycobacterium tuberculosis. Sci Rep. https://doi. org/10.1038/s41598-017-04748-9

Ross AJ (1960) Mycobacterium salmoniphilium sp. nov. from salmonoid fishes. Am Rev Respir Dis 81:241-250. https:// doi.org/10.1164/arrd.1960.81.2.241

Ryndak M, Wang S, Smith I (2008) PhoP, a key player in Mycobacterium tuberculosis virulence. Trends Microbiol

Salimizand H, Jamehdar SA, Nik LB, Sadeghian H (2017) Design of peptides interfering with iron-dependent regulator (IdeR) and evaluation of Mycobacterium tuberculosis growth inhibition. Iran J Basic Med Sci. https://doi.org/10. 22038/ijbms.2017.8859

Seemann T (2014) Prokka: Rapid prokaryotic genome annotation. Bioinformatics 30:2068-2069. https://doi.org/10. 1093/bioinformatics/btu153

Simmon KE, Brown-Elliott BA, Ridge PG et al (2011) Mycobacterium chelonae-abscessus complex associated with sinopulmonary disease, northeastern USA. Emerg Infect Dis 17:1692-1700. https://doi.org/10.3201/eid1709. 101667

Skerman VBD, McGowan VF, Sneath PHA, Peter HA et al (1989) Approved lists of bacterial names. Am Soc Microbiol

Talaat AM, Trucksis M, Kane AS, Reimschuessel R (1999) Pathogenicity of Mycobacterium fortuitum and Mycobacterium smegmatis to goldfish, Carassius auratus. Vet Microbiol 66:151-164

Tatusov RL (2000) The COG database: a tool for genome-scale analysis of protein functions and evolution. Nucleic Acids Res 28:33-36. https://doi.org/10.1093/nar/28.1.33

Van Ingen J, Blaak H, De Beer J et al (2010) Rapidly growing nontuberculous mycobacteria cultured from home tap and shower water. Appl Environ Microbiol 76:6017-6019. https://doi.org/10.1128/AEM.00843-10 
Wang L, Xu M, Southall N et al (2016) A high-throughput assay for developing inhibitors of PhoP, a Virulence Factor of Mycobacterium tuberculosis. Comb Chem High Throughput Screen. https://doi.org/10.2174/ 1386207319666161010163249

Weisburg WG, Barns SM, Pelletier DA, Lane DJ (1991) 16S ribosomal DNA amplification for phylogenetic study. J Bacteriol 173:697-703. https://doi.org/10.1128/jb.173.2. 697-703.1991

Whipps C, Matthews J, Kent M (2008) Distribution and genetic characterization of Mycobacterium chelonae in laboratory zebrafish Danio rerio. Dis Aquat Organ 82:45-54. https:// doi.org/10.3354/dao01967

Whipps CM, Butler WR, Pourahmad F et al (2007) Molecular systematics support the revival of Mycobacterium salmoniphilum (ex Ross 1960) sp. nov., nom. rev., a species closely related to Mycobacterium chelonae. Int J Syst Evol Microbiol 57:2525-2531. https://doi.org/10.1099/ijs.0. 64841-0

Wright ES (2016) Using DECIPHER v2.0 to analyze big biological sequence data in R. R J 8:352-359. V12242009

Wu S, Zhu Z, Fu L et al (2011) WebMGA: A customizable web server for fast metagenomic sequence analysis. BMC Genomics 12:444. https://doi.org/10.1186/1471-2164-12444
Xu L, Dong Z, Fang L et al (2019) OrthoVenn2: a web server for whole-genome comparison and annotation of orthologous clusters across multiple species. Nucleic Acids Res 47:W52-W58. https://doi.org/10.1093/nar/gkz333

Zankari E, Hasman H, Cosentino S et al (2012) Identification of acquired antimicrobial resistance genes. J Antimicrob Chemother. https://doi.org/10.1093/jac/dks261

Zanoni RG, Florio D, Fioravanti ML et al (2008) Occurrence of Mycobacterium spp. in ornamental fish in Italy. J Fish Dis 31:433-441. https://doi.org/10.1111/j.1365-2761.2008. 00924.x

Zerihun MA, Berg V, Lyche JL et al (2011a) Mycobacterium salmoniphilum infection in burbot Lota lota. Dis Aquat Organ 95:57-64. https://doi.org/10.3354/dao02347

Zerihun MA, Nilsen H, Hodneland S, Colquhoun DJ (2011b) Mycobacterium salmoniphilum infection in farmed Atlantic salmon, Salmo salar L. J Fish Dis 34:769-781. https://doi.org/10.1111/j.1365-2761.2011.01293.x

Zondervan NA, Van Dam JCJ, Schaap PJ et al (2018) Regulation of three virulence strategies of Mycobacterium tuberculosis: a success story. Int J Mol Sci

Publisher's Note Springer Nature remains neutral with regard to jurisdictional claims in published maps and institutional affiliations. 